Conclusions The exercise fidelity was low with only three out of five exercises observed performed according to instructions. Only half of the total programme exercises were being executed. Only a third of coaches believed IPEP can reduce injury risk; thus current delivery strategies might be insufficient for translating evidence to this key-stakeholder group. Future work is needed to inform evidence-based strategies to better support the implementation of IPEPs in sport settings.

\section{THE EFFECT OF A WORKSHOP ON COACHES' ADOPTION AND ADHERENCE TO THE ACTIVATE INJURY PREVENTION EXERCISE PROGRAMME}

${ }^{1}$ Craig Barden, ${ }^{1,2}$ Carly McKay, ${ }^{1,3}$ Keith Stokes. 'Department for Health, University of Bath, Bath, BA2 7AY, UK., Bath, UK; ${ }^{2}$ Centre for Motivation and Health Behaviour Change, University of Bath, Bath, BA2 7AY, UK., Bath, UK; ${ }^{3}$ Rugby Football Union, Twickenham, TW2 7BA, UK, London, UK

\subsection{6/bjsports-2021-IOC.20}

Background Educational workshops have been employed in football to positively influence coach perceptions towards injury risk and prevention, and to improve adherence to neuromuscular training programmes. Similar implementation strategies have been used in rugby but their effect on behaviour is unknown.

Objective To assess coaches' perceptions towards injury risk and prevention, and to determine the effect of workshop attendance on their uptake of Activate, a rugby-specific injury prevention exercise programme.

Design Prospective cohort study.

Setting English school rugby union (under-12 to under-18).

Participants School rugby coaches and support staff $(n=54)$.

Interventions All coaches completed a baseline questionnaire investigating their perceptions towards injury risk and prevention. Coaches were invited to attend a pre-season Activate workshop.

Main outcome measures Participants were grouped by workshop attendance (yes/no). Perceptions towards injury risk and prevention were compared at baseline, prior to workshop attendance (Mann-Whitney-U test). Coaches self-reported adoption (programme use; chi-square test) and adherence (sessions completed per week; Mann-Whitney-U test) at postseason.

Results Thirty-three of 54 coaches attended a pre-season workshop. Prior to the workshop, $72 \%$ of coaches were aware of Activate. At baseline, attendees were significantly more likely to believe that exercises shown to prevent injuries should be included in rugby training $(\mathrm{z}=-2.03, \mathrm{p}=0.04)$ and completing a specific warm-up prior to every match would improve players' physical characteristics $(z=-2.13, p=0.03)$. Coaches attending a workshop were significantly more likely to adopt Activate $\left(\chi^{2}=5.05, \mathrm{p}=0.02\right)$, with $17 \%$ greater adherence $(\geq 2$ times per week; $\mathrm{z}=-2.20, \mathrm{p}=0.03)$.

Conclusions Coaches with stronger baseline perceptions regarding injury prevention were more likely to attend a workshop and had significantly greater programme adoption and adherence. If coach perceptions are a determinant of workshop attendance, and subsequently programme uptake, researchers and stakeholders should focus upon strategies to influence coach perceptions towards injury risk and prevention.

\section{CAN A MASSIVE OPEN ONLINE COURSE (MOOC) INFORM CONCUSSION PREVENTION KNOWLEDGE TRANSLATION?}

${ }^{1}$ Stephanie Adams, ${ }^{2}$ Pierre Fremont, ${ }^{3}$ Jennifer Lock, $4,5,6$ Keith 0 Yeates, $1,8,5,9,6,7$ Carolyn Emery, ${ }^{10}$ Leslie Reid, ${ }^{11}$ Penny Werthner, ${ }^{1,4,5,6,12,13}$ Kathryn Schneider. ${ }^{1}$ Sport Injury Prevention Research Centre, Faculty of Kinesiology, University of Calgary, Calgary, Canada; '2Department of Rehabilitation, Faculty of Medicine, Université Laval, Québec, Canada; ${ }^{3}$ Werklund School of Education, University of Calgary, Calgary, Canada; ${ }^{4}$ Department of Psychology, Faculty of Arts, University of Calgary, Calgary, Canada; ${ }^{5}$ Hotchkiss Brain Institute, Cumming School of Medicine, University of Calgary, Calgary, Canada; 'Alberta Children's Hospital Research Institute, Cumming School of Medicine, University of Calgary, Calgary, Canada; ${ }^{7}$ Community Health Sciences, Cumming School of Medicine, University of Calgary, Calgary, Canada; ${ }^{8}$ Department of Pediatrics, Cumming School of Medicine, University of Calgary, Calgary, Canada; ${ }^{9} O^{\prime}$ Brien Institute for Public Health, Cumming School of Medicine, University of Calgary, Calgary, Canada; ${ }^{10}$ Faculty of Science, University of Calgary, Calgary, Canada; ${ }^{11}$ Faculty of Kinesiology, University of Calgary, Calgary, Canada; ${ }^{12}$ Acute Sport Concussion Clinic, Sport Medicine Centre, University of Calgary, Calgary, Canada; ${ }^{13}$ Evidence Sport and Spinal Therapy, Calgary, Canada

\subsection{6/bjsports-2021-IOC.21}

Background To reduce the burden of concussion, effective prevention strategies are required. These should: 1) focus on primary prevention in addition to secondary prevention of recurrence and 2) promote global engagement. A Massive Open Online Course (MOOC) on concussion allows stakeholders to interact with experts while learning about the latest evidence and resources on concussion prevention, and may contribute to enhancing awareness and knowledge of prevention.

Objective To evaluate the feasibility and effectiveness of a MOOC, which includes a focus on concussion prevention, as a global knowledge translation (KT) strategy with multiple stakeholders.

Design De-identified learning analytics were used to assess participation, completion rates, and knowledge gains.

Setting Worldwide web.

Participants Data from 12751 self-registered learners (e.g., health professionals, coaches, sports organisations, athletes, parents, teachers, school administrators) was analysed over 2 iterations of the course.

Intervention The MOOC was available for 9 weeks and included six modules: introduction to concussion, primary prevention, detection (secondary prevention), management, treatment (tertiary prevention) and implementation/revision of protocols. Each module featured interactive learning content/ materials and a reflective process supporting KT and evaluations (for more: https://www.ucalgary.ca/knes/online-concussioncourse).

Main outcome measurements Number of registered learners accessing and completing each module; post-module exam scores; pre-test and post-course exam scores.

Results Of 12751 people who registered, 4195 (33\%) successfully completed all evaluations. The modules were highly accessed by participants [introduction $=7212$ (57\%), prevention=5906 (46\%), detection=5149 (40\%), management $=4716$ $(37 \%)$, treatment $=4468(35 \%)$, protocols $=4241(33 \%)]$. For the first iteration, the prevention module had the highest mean post-module exam score 92.06\% [95\% CI; $91.76-$ 92.36)] and there was a $12 \%$ increase in overall knowledge between pre- and post-course exam scores (mean score increased from $67 \%$ to $79 \%$ ).

Conclusions The sustained engagement of the participants suggests that a MOOC on concussion may be an effective 
and globally accessible KT approach. The MOOC also successfully combined primary prevention with more traditional secondary and tertiary concussion prevention foci, thereby enhancing programming that aims to improve concussion literacy.

\section{IS IT POSSIBLE TO STIMULATE INJURY-PREVENTIVE BEHAVIOUR IN ADULT NOVICE RUNNERS WITH AN ONLINE INTERVENTION? RESULTS OF A RANDOMIZED CONTROLLED TRIAL}

Ellen Kemler, Maaike Cornelissen, Vincent Gouttebarge. Dutch Consumer Safety Institute, Amsterdam, Netherlands

\subsection{6/bjsports-2021-IOC.22}

Background In addition to beneficial health effects, running is associated with a risk of sustaining injuries. The online intervention Runfitcheck was developed to stimulate injury-preventive behaviour among adult novice runners.

Objective To evaluate the effectiveness of Runfitcheck on injury-preventive behaviour among adult novice runners.

Design Randomized controlled trial.

Setting Participants were recruited via Dutch social media networks. Participants were asked to fill in four online questionnaires.

Participants The group of participants consisted of adult novice runners.

Interventions (or Assessment of Risk Factors) The intervention group had access to the Runfitcheck intervention, while the control group performed their running activities as usual.

Main Outcome Measurements Injury-preventive behaviour: (i) using a (personalised) training schedule; (ii) performing strength and technique exercises; and (iii) performing a warmup routine prior to running. Relative Risks ((RR) and 95\% Confidence Interval $(95 \% \mathrm{CI})$ ) were used to analyse behavioural change among runners who were not performing the favourable behaviour at enrolment.

Results A total of 1,411 novice runners $(72.6 \%$ female, mean age 38.1 years) were included. Runners in the intervention group searched more often for information about a warm-up routine $(53.6 \%$ versus $33.6 \%$; $n=194$; RR 1.444 (95\%CI 1.098-1.901)), performed a regular warm-up routine more often $(47.1 \%$ versus $28.4 \% ; n=196 ; R R \quad 1.461 \quad$ (95\%CI 1.084-1.968), and added strength exercises to their warm-up routine more often than runners in the control group (32.6\% versus $17.4 \%$; $n=192$; RR 1.504 (95\%CI 1.0392.179)). Runners in the intervention group performed strength exercises less often at other points of time during the week (other than during their warm-up routine) than runners in the control group $(36.6 \%$ versus $50.0 \% ; n=426$; RR 0.790 (95\%CI 0.669-0.932)). No significant results were found for using a training schedule and running technique exercises.

Conclusions The online intervention Runfitcheck was effective in stimulating aspects of injury-preventive behaviour in adult novice runners related to a warm-up routine, but did not succeed in stimulating other aspects of injury-preventive behaviour.

\section{DIFFERENCES IN INJURY CHARACTERISTICS BETWEEN ATHLETICS DISCIPLINES DURING INTERNATIONAL ATHLETICS CHAMPIONSHIPS}

1,2,3,4,5 Pascal Edouard, 6 Laurent Navarro, ${ }^{5}$ Pedro Branco, 4,7Vincent Gremeaux ${ }^{8}$ Toomas Timpka, ${ }^{9,10}$ Astrid Junge. ${ }^{1}$ Inter-University Laboratory of Human Movement Science (LIBM EA 7424), University of Lyon, University Jean Monnet, Saint-Etienne, France; ${ }^{2}$ Department of Clinical and Exercise Physiology, Sports Medicine Unit, University Hospital of Saint-Etienne, Faculty of Medicine, Saint-Etienne, France; ${ }^{3}$ Medical Commission, French Athletics Federation (FFA), Paris, France; ${ }^{4}$ Swiss Olympic Medical center, Centre de médecine du sport, Division de médecine physique et réadaptation, Centre Hospitalier Universitaire Vaudois, Lausanne, Switzerland; ${ }^{5}$ European Athletics Medical and Anti Doping Commission, European Athletics Association (EAA), Lausanne, Switzerland; ${ }^{6}$ Mines SaintEtienne, Univ Lyon, Univ Jean Monnet, INSERM, U 1059 Sainbiose, Centre CIS, SaintEtienne, France; ${ }^{7}$ Institute of Sport Sciences, University of Lausanne, Lausanne, Switzerland; ${ }^{8}$ Athletics Research Center, Linköping University, Linkoping, Sweden; ${ }^{9}$ Medical School Hamburg, Hamburg, Germany; ${ }^{10}$ Swiss Concussion Center and Schulthess Clinic Zürich, Zurich, Switzerland

\subsection{6/bjsports-2021-IOC.23}

Background Competing in international athletics championships bears the risk of injury. Athletics is composed of different disciplines with different physical, mechanical, technical and psychological demands, leading to different injury rates according to disciplines. However, the specific injury characteristics according to disciplines have not been yet described for high-level athletes during international championships.

Objective To analyse differences in injury characteristics between athletics disciplines during international athletics championships.

Design Prospective study.

Setting 14 international championships from 2007 to 2018.

Participants 8925 male and 7614 female registered international-level athletes.

Main outcome measurements The national medical teams and the local organizing committee physicians reported all newly incurred injuries daily on a standardised injury report form, including the characteristics of each injury: location, type, cause and severity. We analysed differences in injury characteristics distribution between the nine disciplines separately for male and female athletes using $\mathrm{Chi}^{2}$-tests, or Fisher' exact test where appropriate.

Results A total of 928 injuries were reported in male and 597 in female athletes. Injury characteristics significantly varied between disciplines for location, type, cause and severity, in both male and female athletes. Thigh muscle injuries were the main injury diagnoses in sprints, hurdles, jumps, combined events and race walking, lower leg muscle injuries in marathon, lower leg skin injury in middle and long distances, and trunk muscle and lower leg muscle injuries in throws.

Conclusions Short-distance running disciplines exposed to thigh muscle injuries, while long-distance running disciplines exposed to lower leg muscle injuries, and disciplines involving plyometric (i.e. jumps or combined events) are associated with musculoskeletal injuries of different locations and types. Strategies for medical service provision, including local organisation, medical teams, supplies, and facilities, in the preparation of and at athletics championships should be discipline-specific and prepared for targeting the main injuries in each discipline as they appear in the program. 